\title{
Stability and Performance of Continuous-Time Power Control in Wireless Networks
}

\author{
Hamid Reza Feyzmahdavian, Themistoklis Charalambous, and Mikael Johansson
}

\begin{abstract}
This paper develops a comprehensive stability analysis framework for general classes of continuous-time power control algorithms under heterogeneous time-varying delays. Our first set of results establish global asymptotic stability of power control laws involving two-sided scalable interference functions, and include earlier work on standard interference functions as a special case. We then consider contractive interference functions and demonstrate that the associated continuous-time power control laws always have unique fixed points which are exponentially stable, even under bounded heterogeneous time-varying delays. For this class of interference functions, we present explicit bounds on the decay rate that allow us to quantify the impact of delays on the convergence time of the algorithm. When interference functions are linear, we also prove that contractivity is necessary and sufficient for exponential stability of continuous-time power control algorithms with time-varying delays. Finally, numerical simulations illustrate the validity of our theoretical results.
\end{abstract}

Index Terms-Interference functions, positive systems, power control, time-varying delays, wireless networks.

\section{INTRODUCTION}

$\mathbf{S}$ INCE wireless communication devices operate within limited radio spectra, the need to efficiently manage the available spectrum grows as wireless devices become more pervasive. However, wireless communication devices that share the same radio frequency experience $\mathrm{Co}$-Channel Interference (CCI), which impairs the achievable communication rates. A wireless device that unilaterally increases its transmit power can expect to sustain successful transmissions over longer distances and at higher data rates. However, the increased transmit power will increase the co-channel interference seen by other users, and will drain the energy stored in the device battery faster. Adaptive power control algorithms allow devices to setup and maintain wireless links with minimum power while satisfying constraints on Quality of Service (QoS). Such power control approaches are suitable for services with strict QoS requirements, such as voice and video telephony, with prescribed fixed transmission Bit Error Rates (BER). This paper focuses Signalto-Interference-and-Noise Ratio (SINR) as QoS measure; this

Manuscript received November 26, 2012; revised October 5, 2013 and March 23, 2014; accepted March 30, 2014. Date of publication April 2, 2014; date of current version July 21, 2014. This work was supported in part by the Swedish Foundation for Strategic Research (SSF) and the Swedish Science Foundation (VR). Recommended by Associate Editor E. K. P. Chong.

The authors are with the Department of Automatic Control, ACCESS Linnaeus Center, School of Electrical Engineering, KTH-Royal Institute of Technology, SE-10044 Stockholm, Sweden (e-mail: hamidrez@kth.se; themisc@kth.se; mikaelj@kth.se).

Color versions of one or more of the figures in this paper are available online at http://ieeexplore.iee.org.

Digital Object Identifier 10.1109/TAC.2014.2314951 is reasonable, since other relevant measures of QoS, such as the BER, are monotone functions of the SINR.

Initial work on distributed power control with SINR-based QoS was done by Zander [1], assuming a linear model of interference and negligible receiver noise. Zander proposed the Distributed Balancing Algorithm (DBA) in which the power levels of the transmitters constituting the network are updated in a distributed fashion to obtain the greatest Signalto-Interference-Ratio (SIR) that they are capable of jointly achieving. Subsequently, Foschini and Miljanic [2], proposed continuous- and discrete-time power control algorithms (henceforth called Foschini-Miljanic algorithms) for the linear interference model that account for the thermal noise and provide power control with user-specific SINR requirements. These algorithms converge to the optimal power allocation, if one exists, and are based on locally available information only; specifically, the current transmit power level and the measured interference at the receiver of each communication pair are used to update of the power level on that link. If there exist transmit power levels so that the SINR targets on all links can be met, then the algorithm will converge to such a configuration; otherwise, the transmit powers will diverge.

The seminal work by Foschini and Miljanic [2] on continuous- and discrete-time power control triggered off numerous publications for both continuous-time power control (e.g., [3]-[8]) and discrete-time power control (e.g., [9]-[15]). Continuous- and discrete-time power control in wireless networks has been also considered within the context of noncooperative game theory (see for example [16]-[19] and references therein), where each wireless node is assigned a utility as a function of the power it consumes and SINR it attains, and hence dynamically decides its transmission power level so as to maximize its own utility.

An elegant axiomatic framework for studying a general class of discrete-time power control laws, the so-called standard interference functions, was proposed by Yates [10]. This framework includes the discrete-time Foschini-Miljanic (FM) algorithm [2] and several nonlinear power control algorithms as special cases. Monotone convergence of standard interference functions was also obtained by Altman et al. [17] within the context of noncooperative game theory. This framework was later extended by Sung and Leung [11] to a more general class, the so-called two-sided scalable interference functions, which includes the standard interference functions as a special case, and allows for a simple and powerful analysis of certain opportunistic power control laws that the standard framework cannot handle. While these frameworks are general and guarantee synchronous and asynchronous convergence of discrete- 
time power control algorithms in their class, the existence of fixed points has to be established separately, and there is no information about the convergence rate of the algorithms. Recently, Feyzmahdavian et al. [15] explored the connections between the standard interference function framework and the theory for fixed-point iterations. They showed that standard interference functions do, in general, not define contraction mappings and introduced a new framework, called contractive interference functions, that guarantees existence and uniqueness of fixed-point along with linear convergence of iterates. They also demonstrated that several important distributed power control algorithms proposed in the literature are contractive and derived the associated convergence rates.

The continuous-time power control, apart from its motivational aspects, offers a simple model that can be used for future studies on high-level wireless systems. For example, while in the literature it is assumed that power control takes place in discrete time intervals (and can only be used as long as the time duration for successive adjustments of transmitter powers is less than the coherence time of the channel), in continuoustime power control there is no restriction on how fast the wireless channel is varying (see, e.g., [3]). While communication delays are inevitably omnipresent in networks, their impact on stability of continuous-time power control laws have not been considered until recently. Charalambous et al. [5] used the multivariate Nyquist criterion to show that the continuous-time FM algorithm is asymptotically stable for arbitrary constant time-delays, while Zappavinga et al. [7] used theory for positive linear systems to prove that the FM algorithm is asymptotically stable even in the presence of bounded time-varying communication delays and topology changes. The continuoustime counterpart of the standard interference functions firstly appeared in [4] and it was later analyzed in [8] using LyapunovRazumikhin approach.

In this paper, we consider general classes of continuous-time distributed power control algorithms, which are continuoustime versions of those proposed in [11] and [15]. We analyze the continuous-time two-sided scalable interference functions (for which standard interference functions constitute a special case) and contractive interference functions. More specifically, we make the following contributions. Firstly, we prove that if there exists a feasible steady state power vector, power-control laws involving two-sided scalable interference functions are asymptotically stable for arbitrary bounded heterogeneous time-varying delays. Next, for the contractive interference functions, we prove that associated continuous-time power control algorithms converge exponentially to the unique fixed points, even in the presence of bounded communication delays. For this class of interference functions, we derive explicit bounds on the decay rate, thus quantifying the impact of delays on the convergence time of the algorithm. When interference functions are linear, we also show that contractivity is necessary and sufficient for exponential stability of continuous-time power control algorithms with time-varying delays. Finally, the validity of our theoretical results is demonstrated via illustrative examples for the continuous-time FM algorithm [2] and the continuous-time version of the Utility-Based Power Control (UBPC) algorithm proposed by Xiao et al. [20]. The improved understanding of the continuous-time power control that we develop can potentially be used in various stochastic analysis frameworks that rely on ODE-limits (e.g., mean-field games and stochastic approximation).

The remainder of the paper is organized as follows. In Section II, we introduce the notation that will be used and review some preliminaries that are useful for the development of the results in this paper. Section III gives a description of the problem under consideration, while our main results for twosided scalable, contractive and linear interference functions are stated in Sections IV, V and VI, respectively. Illustrative examples are presented in Section VII, justifying the validity of our results. Finally, concluding remarks are given in Section VIII.

\section{Notation AND PRELIMINARIES}

\section{A. Notation}

Throughout the paper, vectors are written in bold lower case letters and matrices in capital letters. We let $\mathbb{R}, \mathbb{N}$, and $\mathbb{N}_{0}$ denote the set of real numbers, natural numbers, and the set of natural numbers including zero, respectively. The non-negative orthant of the $n$-dimensional real space $\mathbb{R}^{n}$ is represented by $\mathbb{R}_{+}^{n}$. The $i^{t h}$ component of a vector $\boldsymbol{x} \in \mathbb{R}^{n}$ is denoted by $x_{i}$, and the notation $\boldsymbol{x} \geq \boldsymbol{y}$ means that $x_{i} \geq y_{i}$ for all components $i$. Given a vector $\boldsymbol{v}>\mathbf{0}$, the weighted $l_{\infty}$ norm is defined by

$$
\|\boldsymbol{x}\|_{\infty}^{\boldsymbol{v}}=\max _{1 \leq i \leq n} \frac{\left|x_{i}\right|}{v_{i}}
$$

For a matrix $A \in \mathbb{R}^{n \times n}, a_{i j}$ denotes the entry in row $i$ and column $j$. The spectral radius of a matrix $A$ is the largest magnitude of its eigenvalues and is denoted by $\rho(A)$. The function $\operatorname{sgn}(x)$ is the signum function defined by

$$
\operatorname{sgn}(x)= \begin{cases}1, & \text { if } x>0, \\ 0, & \text { if } x=0, \\ -1, & \text { if } x<0\end{cases}
$$

The upper-right Dini-derivative of a function $h: \mathbb{R} \rightarrow \mathbb{R}$ is denoted by $D^{+} h(\cdot)$. For a given $\tau_{\max }>0, \mathcal{C}$ denotes the space of all real-valued continuous functions on $\left[-\tau_{\max }, 0\right]$ taking values in $\mathbb{R}^{n}$. We also denote

$$
\mathcal{C}_{++}=\left\{\varphi(\cdot) \in \mathcal{C} \mid \varphi(\theta)>\mathbf{0}, \theta \in\left[-\tau_{\max }, 0\right]\right\} .
$$

\section{B. Preliminaries}

Next, we review the key definitions and results necessary for developing the main results of this paper. We start with the definition of non-negative and Metzler matrices.

Definition 1: A matrix $A \in \mathbb{R}^{n \times n}$ is said to be non-negative if $a_{i j} \geq 0$ for all $i$ and $j$. It is called a Metzler matrix if $a_{i j} \geq 0$ for all $i \neq j$.

The next proposition provides some known properties of non-negative matrices. These conditions are useful when analyzing the stability of power control algorithms involving linear interference functions.

Proposition 1 ([21, Ch. 6]): Let $A \in \mathbb{R}^{n \times n}$ be non-negative. Then, the following statements are equivalent.

(a) There exists a vector $\boldsymbol{v}>\mathbf{0}$ such that $A \boldsymbol{v}<\boldsymbol{v}$. 
(b) There exists a vector $\boldsymbol{v}>\mathbf{0}$ such that $\|A\|_{\infty}^{\boldsymbol{v}}<1$.

(c) $\rho(A)<1$.

For nonlinear power control laws, our analysis relies on contraction mappings, defined next.

Definition 2: A mapping $f: \mathbb{R}^{n} \rightarrow \mathbb{R}^{n}$ is called a ccontraction if there exists a constant $c \in[0,1)$ such that

$$
\|\boldsymbol{f}(\boldsymbol{x})-\boldsymbol{f}(\boldsymbol{y})\| \leq c\|\boldsymbol{x}-\boldsymbol{y}\|, \quad \forall x, y \in \mathbb{R}^{n},
$$

where $\|\cdot\|$ is some norm on $\mathbb{R}^{n}$.

An attractive feature of contraction mappings is that they always have a unique fixed point.

Proposition 2 ([22, Ch. 3]): If $f: \mathbb{R}^{n} \rightarrow \mathbb{R}^{n}$ is a ccontraction, then $f$ has a unique fixed point, i.e.,

$$
\exists \boldsymbol{x}^{\star} \in \mathbb{R}^{n} \text { such that } \boldsymbol{f}\left(\boldsymbol{x}^{\star}\right)=\boldsymbol{x}^{\star} .
$$

The following proposition helps us to study the exponential stability of continuous-time power control algorithms in the presence of bounded time-varying delays.

Proposition 3 ([23, pp. 389-390]): Let $\tau_{\max }, \alpha$ and $\beta$ be constants with $0<\alpha<\beta$, and $\tau_{\max } \geq 0$. If a continuous nonnegative function $y(t)$ satisfies

$$
\dot{y}(t) \leq-\beta y(t)+\alpha\left(\sup _{t-\tau_{\max } \leq s \leq t} y(s)\right), \quad t \geq 0
$$

then

$$
y(t) \leq\left(\sup _{-\tau_{\max } \leq s \leq 0} y(s)\right) e^{-\eta t}
$$

holds for all $t \geq 0$, where $\eta$ is the unique positive solution to

$$
\eta=\beta-\alpha e^{\eta \tau_{\max }}
$$

\section{Problem Statement}

We consider a wireless network consisting of $n$ mobile users communicating over the same frequency band. The continuoustime power control algorithm, firstly considered in its general form by [4], is given by

$$
\frac{\mathrm{d} p_{i}(t)}{\mathrm{d} t}=k_{i}\left(-p_{i}(t)+I_{i}(\boldsymbol{p}(t))\right), \quad i=1, \ldots, n .
$$

Here, $p_{i}(t)$ is the transmitted power of user $i$ at time $t, I_{i}$ : $\mathbb{R}_{+}^{n} \mapsto \mathbb{R}_{+}$is the interference function modeling the interference and noise experienced by the intended receiver of user $i$, and $k_{i}$ is a positive constant representing the proportionality constant. The well-known continuous-time Foschini-Miljanic algorithm [2], for example, considers the linear interference function given by:

$$
I_{i}(\boldsymbol{p})=\gamma_{i} \frac{\sum_{j \neq i} g_{i j} p_{j}+\eta_{i}}{g_{i i}}, \quad i=1, \ldots, n
$$

where $g_{i j} \geq 0$ is the channel gain on the link between user $j$ and the receiver of user $i, \gamma_{i}$ is the target Signal-to-Interference-andNoise Ratio (SINR) of user $i$, and $\eta_{i}$ is the background noise at the receiver of user $i$.
In practice, there will always be a signaling delay associated with transmitting the perceived interference at the transmitter to the receiver, so that it can adjust the power according to the power control law. Consequently, a realistic analysis of the continuous-time power control algorithm must consider heterogeneous time-varying delays. More precisely, the continuoustime power control algorithm (1), when the time delay is introduced becomes

$$
\begin{cases}\frac{\mathrm{d} p_{i}(t)}{\mathrm{d} t}=k_{i}\left(-p_{i}(t)+I_{i}\left(\boldsymbol{p}^{d_{i}}(t)\right)\right), & t \geq 0 \\ p_{i}(t)=\varphi_{i}(t), & t \in\left[-\tau_{\max }, 0\right]\end{cases}
$$

Here, $\tau_{\max } \geq 0, \varphi(\cdot)=\left[\varphi_{1}(\cdot), \ldots, \varphi_{n}(\cdot)\right]^{T} \in \mathcal{C}$ is the vectorvalued function specifying the initial condition of the system, and

$$
\boldsymbol{p}^{d_{i}}(t)=\left[p_{1}\left(t-\tau_{1}^{i}(t)\right), \ldots, p_{n}\left(t-\tau_{n}^{i}(t)\right)\right]^{T} .
$$

The delays $\tau_{j}^{i}(t)$ are assumed to be time-varying continuous functions with respect to time and satisfy

$$
0 \leq \tau_{j}^{i}(t) \leq \tau_{\max }
$$

for all $i$ and $j$ and all $t \geq 0$. Note that $\tau_{j}^{i}(t)$ are not necessarily continuously differentiable and no restriction on their derivative (such as $\dot{\tau}(t)<1$ ) is imposed.

To put our work in context, note that the discrete-time analog of (1) consists of iterations of the type

$$
\boldsymbol{p}(t+1)=\boldsymbol{I}(\boldsymbol{p}(t)), \quad t \in \mathbb{N}_{0} .
$$

Such iterations have been studied under three frameworks; standard interference functions [10], two-sided scalable interference functions [11], and contractive interference functions [15]. Specifically, it was shown that if $\boldsymbol{I}(\boldsymbol{p})$ is standard or two-sided scalable, and a power vector $\boldsymbol{p}^{\star}>\mathbf{0}$ satisfying $\boldsymbol{p}^{\star}=$ $\boldsymbol{I}\left(\boldsymbol{p}^{\star}\right)$ exists, then the iterates generated by (4) converge asymptotically to $\boldsymbol{p}^{\star}$ [11, Th. 10]. If $\boldsymbol{I}(\boldsymbol{p})$ is contractive, then the iterates (4) converges to a unique fixed point at a linear rate [15, Th. 1], i.e., the distance between the iterates and the fixed point decays exponentially.

The aim of this paper is to develop a stability analysis framework for continuous-time power control algorithms that is equally comprehensive as the theory for its discrete-time counterpart. Our main objectives are therefore to $(i)$ study the asymptotic stability of the continuous-time power control algorithm described by (1) (without delays) and (3) (under bounded heterogeneous time-varying delays) when the interference function $\boldsymbol{I}(\boldsymbol{p})$ is two-sided scalable; and to $(i i)$ analyse the exponential stability of (1) when the interference function is contractive and determine how the convergence rate of (3) depends on the magnitude of the time delays.

\section{STANDARD AND TWO-SIDED SCALABLE INTERFERENCE FUNCTIONS}

As observed by Yates, many interference functions share important properties that allow them to be analyzed in a common framework. This observation led to the definition of standard interference functions. 
Definition 3 ([10]): A function $I: \mathbb{R}_{+}^{n} \rightarrow \mathbb{R}_{+}^{n}$ is called a standard interference function, if for all $\boldsymbol{p} \geq \mathbf{0}$, the following properties are satisfied:

- Positivity: $\boldsymbol{I}(\boldsymbol{p})>\mathbf{0}$.

- Monotonicity: If $\boldsymbol{p} \geq \boldsymbol{p}^{\prime}$, then $\boldsymbol{I}(\boldsymbol{p}) \geq \boldsymbol{I}\left(\boldsymbol{p}^{\prime}\right)$.

- Scalability: For all $\alpha>1, \alpha \boldsymbol{I}(\boldsymbol{p})>\boldsymbol{I}(\alpha \boldsymbol{p})$.

The monotonicity property of standard interference functions requires a user to increase its transmit power when other users increase their powers. Opportunistic power control algorithms, however, work in the opposite way as they allow users with large channel gain and low interference to transmit at high power while they limit users with poor channels and high interference to transmit at low (or even zero) power [24]-[26]. In this way, opportunistic algorithms exploit fluctuations in the channel gains to transmit when the quality of communication links is favorable. Since opportunistic power control algorithms violate the monotonicity condition, they cannot be handled by the standard interference function framework. However, in certain cases they can be analyzed using the following class of generalized interference functions, introduced by Sung and Leung [11]:

Definition 4 ([11]): A function $I: \mathbb{R}_{+}^{n} \rightarrow \mathbb{R}_{+}^{n}$ is called a two-sided scalable interference function, if for all $p \geq 0, I(p)$ satisfies

- Positivity: $\boldsymbol{I}(\boldsymbol{p})>\mathbf{0}$.

- Two-sided scalability: For all $\alpha>1$,

$$
\frac{1}{\alpha} \boldsymbol{p} \leq \boldsymbol{p}^{\prime} \leq \alpha \boldsymbol{p} \Rightarrow \frac{1}{\alpha} \boldsymbol{I}(\boldsymbol{p})<\boldsymbol{I}\left(\boldsymbol{p}^{\prime}\right)<\alpha \boldsymbol{I}(\boldsymbol{p}) .
$$

Even though the monotonicity and scalability conditions of standard interference functions have been replaced by the twosided scalability condition, one can show that every standard interference function is also two-sided scalable [11, Proposition 4]. The following example shows that the converse is, in general, not true. Consider the interference function

$$
I(p)= \begin{cases}\sqrt{p}+\frac{1}{100}, & 0 \leq p \leq 1 \\ \frac{1}{\sqrt{p}}+\frac{1}{100}, & p>1\end{cases}
$$

which is two-sided scalable. However, monotonicity property does not hold and, hence, the interference function is not standard.

The main properties of two-sided scalable interference functions can be summarized as follows.

Proposition 4 ([11], [13]): Let $I: \mathbb{R}_{+}^{n} \rightarrow \mathbb{R}_{+}^{n}$ be a two-sided scalable interference function. Then,

(a) $\boldsymbol{I}(\boldsymbol{p})$ is continuous at $\boldsymbol{p}$ for all $\boldsymbol{p}>\mathbf{0}$.

(b) If $\boldsymbol{I}(\boldsymbol{p})$ has a fixed point, then that fixed point is unique.

(c) If $\boldsymbol{p} \neq \boldsymbol{p}^{\prime}$, then $d\left(\boldsymbol{I}(\boldsymbol{p}), \boldsymbol{I}\left(\boldsymbol{p}^{\prime}\right)\right)<d\left(\boldsymbol{p}, \boldsymbol{p}^{\prime}\right)$, where the distance function $d(\cdot, \cdot)$ is defined as

$$
d\left(\boldsymbol{p}, \boldsymbol{p}^{\prime}\right)=\max _{1 \leq i \leq n}\left\{\max \left\{\frac{p_{i}}{p_{i}^{\prime}}, \frac{p_{i}^{\prime}}{p_{i}}\right\}\right\} .
$$

We begin by studying the asymptotic stability of (1) with $\boldsymbol{I}(\boldsymbol{p})$ being a two-sided scalable interference function. Thanks to $k_{i}$ being a positive constant and $I_{i}(\boldsymbol{p})$ being positive on $\mathbb{R}_{+}^{n}$, differential equations (1) and (3) define positive systems ${ }^{1}$ [27, Ch. 3]. Therefore, the physical constraint that the power should be non-negative $\left(p_{i}(t) \geq 0\right)$ is automatically fulfilled. The following theorem is our first key result, which shows that if a two-sided scalable interference function $\boldsymbol{I}(\boldsymbol{p})$ has a fixed point $\boldsymbol{p}^{\star}>\mathbf{0}$, then the solution to (1) converges asymptotically to $\boldsymbol{p}^{\star}$ for any positive initial condition $\boldsymbol{p}(0)$.

Theorem 1 (Nominal Power Control Algorithm): If a twosided scalable interference function $I: \mathbb{R}_{+}^{n} \rightarrow \mathbb{R}_{+}^{n}$ has a fixed point $p^{\star}>\mathbf{0}$, then the continuous-time power control algorithm (1) is asymptotically stable for any initial condition $\boldsymbol{p}(0)>\mathbf{0}$, and for any proportionality constant $k_{i}>0$.

Proof: Let $p^{\star}$ be the fixed point of the interference function $\boldsymbol{I}(\boldsymbol{p})$, i.e., $\boldsymbol{p}^{\star}=\boldsymbol{I}\left(\boldsymbol{p}^{\star}\right)$, and let $K=\operatorname{diag}\left(k_{1}, \ldots, k_{n}\right)$. It follows from Lagrange's formula [28, p. 242] that the solution $\boldsymbol{p}(t)$ to (1) is given by

$$
\boldsymbol{p}(t)=e^{-K t} \boldsymbol{p}(0)+\int_{0}^{t} e^{-K(t-s)} K \boldsymbol{I}(\boldsymbol{p}(s)) \mathrm{ds}, \quad t \geq 0
$$

where $e^{-K t}=\operatorname{diag}\left(e^{-k_{1} t}, \ldots, e^{-k_{n} t}\right)$. Since the initial condition $\boldsymbol{p}(0)$ is assumed to be positive and $\boldsymbol{I}(\boldsymbol{p})>\mathbf{0}$ for all $\boldsymbol{p} \geq \mathbf{0}$, it follows that $\boldsymbol{p}(t)>\mathbf{0}$ for all $t \geq 0$ [27]. This allows us to use the Lyapunov function

$$
V(\boldsymbol{p}(t))=d\left(\boldsymbol{p}(t), \boldsymbol{p}^{\star}\right)-1,
$$

where $d(\cdot, \cdot)$ is defined in (5). It is clear that $V(\boldsymbol{p})>0$ for all $\boldsymbol{p} \neq \boldsymbol{p}^{\star}$. Let $m \in\{1, \ldots, n\}$ be an index such that

$$
m=\underset{1 \leq i \leq n}{\arg \max }\left\{\max \left\{\frac{p_{i}(t)}{p_{i}^{\star}}, \frac{p_{i}^{\star}}{p_{i}(t)}\right\}\right\} .
$$

This together with the definition of $d(\cdot, \cdot)$ implies that

$$
V(\boldsymbol{p}(t))=\max \left\{\frac{p_{m}(t)}{p_{m}^{\star}}, \frac{p_{m}^{\star}}{p_{m}(t)}\right\}-1 .
$$

The upper-right Dini-derivative of $V(\boldsymbol{p}(t))$ along the trajectories of (1) is given by

$$
D^{+} V(\boldsymbol{p}(t))= \begin{cases}\frac{\dot{p}_{m}(t)}{p_{m}^{\star}}, & p_{m}(t)>p_{m}^{\star}, \\ 0, & p_{m}(t)=p_{m}^{\star}, \\ -\frac{p_{m}^{\star}}{p_{m}^{2}}(t) \dot{p}_{m}(t), & p_{m}(t)<p_{m}^{\star} .\end{cases}
$$

Case 1) If $p_{m}(t)>p_{m}^{\star}$, then

$$
\begin{aligned}
D^{+} V(\boldsymbol{p}(t)) & =k_{m}\left(\frac{-p_{m}(t)+I_{m}(\boldsymbol{p}(t))}{p_{m}^{\star}}\right) \\
& =k_{m}\left(-\frac{p_{m}(t)}{p_{m}^{\star}}+\frac{I_{m}(\boldsymbol{p}(t))}{I_{m}\left(\boldsymbol{p}^{\star}\right)}\right)
\end{aligned}
$$

\footnotetext{
${ }^{1}$ A dynamical system is said to be positive if any trajectory of the system starting from non-negative initial conditions remains forever in the positive orthant.
} 
where the second equality holds, since $p_{m}^{\star}=I_{m}\left(\boldsymbol{p}^{\star}\right)$. From the definition of $d(\cdot, \cdot)$, we have

$$
\frac{I_{m}(\boldsymbol{p}(t))}{I_{m}\left(\boldsymbol{p}^{\star}\right)} \leq d\left(\boldsymbol{I}(\boldsymbol{p}(t)), \boldsymbol{I}\left(\boldsymbol{p}^{\star}\right)\right) .
$$

Moreover, $d\left(\boldsymbol{p}(t), \boldsymbol{p}^{\star}\right)=p_{m}(t) / p_{m}^{\star}$, since $p_{m}(t)>p_{m}^{\star}$. Thus

$$
D^{+} V(\boldsymbol{p}(t)) \leq k_{m}\left(-d\left(\boldsymbol{p}(t), \boldsymbol{p}^{\star}\right)+d\left(\boldsymbol{I}(\boldsymbol{p}(t)), \boldsymbol{I}\left(\boldsymbol{p}^{\star}\right)\right)\right) .
$$

Case 2) Similarly, if $p_{m}(t)<p_{m}^{\star}$, then

$$
\begin{aligned}
D^{+} & V(\boldsymbol{p}(t)) \\
& =-k_{m} \frac{p_{m}^{\star}}{p_{m}^{2}(t)}\left(-p_{m}(t)+I_{m}(\boldsymbol{p}(t))\right) \\
& =k_{m} \frac{I_{m}(\boldsymbol{p}(t))}{p_{m}(t)}\left(-\frac{p_{m}^{\star}}{p_{m}(t)}+\frac{I_{m}\left(\boldsymbol{p}^{\star}\right)}{I_{m}(\boldsymbol{p}(t))}\right) \\
& \leq k_{m} \frac{I_{m}(\boldsymbol{p}(t))}{p_{m}(t)}\left(-d\left(\boldsymbol{p}(t), \boldsymbol{p}^{\star}\right)+d\left(\boldsymbol{I}(\boldsymbol{p}(t)), \boldsymbol{I}\left(\boldsymbol{p}^{\star}\right)\right) .\right.
\end{aligned}
$$

By Proposition Proposition 4(c), $d\left(\boldsymbol{I}(\boldsymbol{p}(t)), \boldsymbol{I}\left(\boldsymbol{p}^{\star}\right)\right)<$ $d\left(\boldsymbol{p}(t), \boldsymbol{p}^{\star}\right)$ for $\boldsymbol{p}(t) \neq \boldsymbol{p}^{\star}$. It follows from (6) and (7) that

$$
D^{+} V(\boldsymbol{p}(t))<0
$$

for all $\boldsymbol{p}(t) \neq \boldsymbol{p}^{\star}$. This proves that the fixed point $\boldsymbol{p}^{\star}$ is globally asymptotically stable.

Remark 1: For the particular case when the interference function is standard, Theorem 1 recovers a very recent result in [8].

The next result proves that continuous-time power control laws involving two-sided scalable interference functions still converge asymptotically to the fixed point (provided that one exists), when arbitrary bounded heterogeneous time-varying delays are introduced to the interference function $\boldsymbol{I}(\boldsymbol{p})$.

Theorem (Delayed Power Control Algorithm): If a two-sided scalable interference function $I: \mathbb{R}_{+}^{n} \rightarrow \mathbb{R}_{+}^{n}$ has a fixed point $\boldsymbol{p}^{\star}>\mathbf{0}$, then the continuous-time power control algorithm (3) is asymptotically stable for any initial condition $\varphi(\cdot) \in \mathcal{C}_{++}$, and for any proportionality constant $k_{i}>0$.

Proof: Consider the candidate Lyapunov function

$$
V(\boldsymbol{p}(t))=d\left(\boldsymbol{p}(t), \boldsymbol{p}^{\star}\right)-1 .
$$

As in the proof of Theorem 1, we have

$$
D^{+} V(\boldsymbol{p}(t))= \begin{cases}\frac{\dot{p}_{m}(t)}{p_{m}^{\star}}, & p_{m}(t)>p_{m}^{\star}, \\ 0, & p_{m}(t)=p_{m}^{\star}, \\ -\frac{p_{m}^{\star}}{p_{m}^{2}(t)} \dot{p}_{m}(t), & p_{m}(t)<p_{m}^{\star}\end{cases}
$$

where the index $m$ satisfies

$$
\max \left\{\frac{p_{i}(t)}{p_{i}^{\star}}, \frac{p_{i}^{\star}}{p_{i}(t)}\right\} \leq \max \left\{\frac{p_{m}(t)}{p_{m}^{\star}}, \frac{p_{m}^{\star}}{p_{m}(t)}\right\}
$$

for all $i \in\{1, \ldots, n\}$.
Case 1) If $p_{m}(t)>p_{m}^{\star}$, then

$$
\begin{aligned}
D^{+} & V(\boldsymbol{p}(t)) \\
& =k_{m}\left(-\frac{p_{m}(t)+I_{m}\left(\boldsymbol{p}^{d_{m}}(t)\right)}{p_{m}^{\star}}\right) \\
& =k_{m}\left(-\frac{p_{m}(t)}{p_{m}^{\star}}+\frac{I_{m}\left(\boldsymbol{p}^{d_{m}}(t)\right)}{I_{m}\left(\boldsymbol{p}^{\star}\right)}\right), \\
& \leq k_{m}\left(-d\left(\boldsymbol{p}(t), \boldsymbol{p}^{\star}\right)+d\left(\boldsymbol{I}\left(\boldsymbol{p}^{d_{m}}(t)\right), \boldsymbol{I}\left(\boldsymbol{p}^{\star}\right)\right)\right) .
\end{aligned}
$$

On the other hand, by Proposition 4(c), we obtain

$$
\begin{aligned}
d & (\boldsymbol{I} \\
& \left.\left.<\boldsymbol{p}^{d_{m}}(t)\right), \boldsymbol{I}\left(\boldsymbol{p}^{\star}\right)\right) \\
& <d\left(\boldsymbol{p}^{d_{m}}(t), \boldsymbol{p}^{\star}\right) \\
& =\max _{1 \leq i \leq n}\left\{\max \left\{\frac{p_{i}\left(t-\tau_{i}^{m}(t)\right)}{p_{i}^{\star}}, \frac{p_{i}^{\star}}{p_{i}\left(t-\tau_{i}^{m}(t)\right)}\right\}\right\} \\
& \leq \max _{1 \leq i \leq n} d\left(\boldsymbol{p}\left(t-\tau_{i}^{m}(t)\right), \boldsymbol{p}^{\star}\right) \\
& \leq \max _{t-\tau_{\max } \leq s \leq t} d\left(\boldsymbol{p}(s), \boldsymbol{p}^{\star}\right)
\end{aligned}
$$

where the last inequality holds, since $\tau_{i}^{m}(t) \leq \tau_{\max }$ for all $i$. Substituting (9) into (8) gives

$$
\begin{aligned}
D^{+} V(\boldsymbol{p}(t)) & <k_{m}\left(-d\left(\boldsymbol{p}(t), \boldsymbol{p}^{\star}\right)+\max _{t-\tau_{\max \leq s \leq t}} d\left(\boldsymbol{p}(s), \boldsymbol{p}^{\star}\right)\right) \\
& =k_{m}\left(-V(\boldsymbol{p}(t))+\max _{t-\tau_{\max } \leq s \leq t} V(\boldsymbol{p}(s))\right) .
\end{aligned}
$$

Case 2) Similarly, if $p_{m}(t)<p_{m}^{\star}$, we have

$$
\begin{aligned}
& D^{+} V(\boldsymbol{p}(t)) \\
& \quad \leq k_{m} \frac{I_{m}(\boldsymbol{p}(t))}{p_{m}(t)}\left(-d\left(\boldsymbol{p}(t), \boldsymbol{p}^{\star}\right)+d\left(\boldsymbol{I}\left(\boldsymbol{p}^{d_{m}}(t)\right), \boldsymbol{I}\left(\boldsymbol{p}^{\star}\right)\right)\right) \\
& \quad<k_{m} \frac{I_{m}(\boldsymbol{p}(t))}{p_{m}(t)}\left(-V(\boldsymbol{p}(t))+\max _{t-\tau_{\max } \leq s \leq t} V(\boldsymbol{p}(s))\right) .
\end{aligned}
$$

In order to address the asymptotic stability of (3), we now use the Invariance-Principle theorem for delay differential equations [29, Corollary 1], [30, Corollary 3.3.2]. According to this theorem, since $V\left(\boldsymbol{p}^{\star}\right)=0$ and $V(\boldsymbol{p})>0$ for all $\boldsymbol{p} \in$ $\mathbb{R}_{+}^{n}-\left\{\boldsymbol{p}^{\star}\right\}$, the continuous-time power control algorithm (3) is asymptotically stable for any $\varphi(\cdot) \in \mathcal{C}_{++}$if $D^{+} V(\boldsymbol{p}(t))<0$ whenever

$$
\max _{t-\tau_{\max } \leq s \leq t} V(\boldsymbol{p}(s))=V(\boldsymbol{p}(t)) .
$$

It follows from (10) and (11) that whenever the above condition holds, $D^{+} V(\boldsymbol{p}(t))<0$. Hence, $\boldsymbol{p}=\boldsymbol{p}^{\star}$ is globally asymptotically stable for all $\varphi(\cdot) \in \mathcal{C}_{++}$.

Remark 2: The delay independence of continuous-time power control with standard interference functions was previously considered in [8, Th. 4], using a Lyapunov-Razumikhin approach. Since every standard interference function is also two-sided scalable, Theorem 2 recovers the delay independence of standard interference functions as a special case. Note, however, that our proof differs significantly from the analysis in [8]. The stability proof for standard interference functions in [8] made heavy use of the fact that standard interference 
functions are monotonic, which can not be assumed for twosided scalable interference functions (cf. Definitions 3 and 4).

Remark 3: We have shown that continuous-time power control with two-sided scalable interference functions converges asymptotically, but have not provided any guarantees on convergence rates. This is not due to a conservative analysis, since there are two-sided scalable interference functions for which (1) may converge arbitrarily slowly, see [15, Example 2].

\section{Contractive Interference Functions}

Two-sided scalable interference functions do not necessarily have fixed points in the positive orthant (consider for example $I(p)=p+1)$, and the existence of fixed points has to be verified separately. Furthermore, no guarantees on the convergence rate of continuous-time power control algorithms involving two-sided scalable interference functions are given. In [15], contractive interference functions, a slight modification of the standard interference functions, were introduced to allow for a more powerful analysis of distributed power control algorithms.

Definition 5 ([15]): A function $I: \mathbb{R}_{+}^{n} \rightarrow \mathbb{R}_{+}^{n}$ is said to be a c-contractive interference function if for all $\boldsymbol{p} \geq \mathbf{0}$, it satisfies the following conditions:

- Positivity: $\boldsymbol{I}(\boldsymbol{p})>\mathbf{0}$.

- Monotonicity: If $\boldsymbol{p} \geq \boldsymbol{p}^{\prime}$, then $\boldsymbol{I}(\boldsymbol{p}) \geq \boldsymbol{I}\left(\boldsymbol{p}^{\prime}\right)$.

- Contractivity: There exists a constant $c \in[0,1)$, and a vector $\boldsymbol{v}>\mathbf{0}$ such that for all $\epsilon>0$,

$$
\boldsymbol{I}(\boldsymbol{p}+\epsilon \boldsymbol{v}) \leq \boldsymbol{I}(\boldsymbol{p})+c \epsilon \boldsymbol{v} .
$$

Contrary to the result for standard and two-sided scalable interference functions, contractive interference functions define contraction mappings in the weighted $l_{\infty}$ norm, which implies that $\boldsymbol{I}(\boldsymbol{p})=\boldsymbol{p}$ always admits a unique solution in $\mathbb{R}_{+}^{n}$.

Proposition 5 ([15]): If $I: \mathbb{R}_{+}^{n} \rightarrow \mathbb{R}_{+}^{n}$ is a c-contractive interference function, then it has a unique fixed point $\boldsymbol{p}^{\star} \in \mathbb{R}_{+}^{n}$, and

$$
\left\|\boldsymbol{I}(\boldsymbol{p})-\boldsymbol{I}\left(\boldsymbol{p}^{\prime}\right)\right\|_{\infty}^{\boldsymbol{v}} \leq c\left\|\boldsymbol{p}-\boldsymbol{p}^{\prime}\right\|_{\infty}^{\boldsymbol{v}}, \quad \forall \boldsymbol{p}, \boldsymbol{p}^{\prime} \in \mathbb{R}_{+}^{n} .
$$

In [15], it was shown that the linear interference function (2), as well as many practical interference functions from the literature are contractive. In particular, all the examples provided in Yates's original paper [10] were shown to be contractive.

We will now show that if the interference function is ccontractive, then the continuous-time power control law described by (1) converges exponentially to the unique fixed point. Note that contractive interference functions are also positive and hence, the physical constraint that the power should be nonnegative $\left(p_{i}(t) \geq 0\right)$ is fulfilled.

Theorem 3 (Nominal Power Control Algorithm): If an interference function $I: \mathbb{R}_{+}^{n} \rightarrow \mathbb{R}_{+}^{n}$ is c-contractive, then the continuous-time power control algorithm (1) is exponentially stable for any initial condition $\boldsymbol{p}(0)>\mathbf{0}$, and for any proportionality constant $k_{i}>0$. In particular, the solution $\boldsymbol{p}(t)$ of (1) satisfies

$$
\left\|\boldsymbol{p}(t)-\boldsymbol{p}^{\star}\right\|_{\infty}^{\boldsymbol{v}} \leq\left\|\boldsymbol{p}(0)-\boldsymbol{p}^{\star}\right\|_{\infty}^{\boldsymbol{v}} e^{-k_{\min }(1-c) t}, \quad t \geq 0,
$$

where $k_{\min }=\min _{1 \leq i \leq n} k_{i}$.
Proof: Consider the Lyapunov function

$$
V(\boldsymbol{p}(t))=\left\|\boldsymbol{p}(t)-\boldsymbol{p}^{\star}\right\|_{\infty}^{\boldsymbol{v}} .
$$

It follows from the definition of the weighted $l_{\infty}$ norm that

$$
V(\boldsymbol{p}(t))=\frac{1}{v_{m}}\left|p_{m}(t)-p_{m}^{\star}\right|,
$$

where the index $m \in\{1, \ldots, n\}$ satisfies

$$
\frac{1}{v_{i}}\left|p_{i}(t)-p_{i}^{\star}\right| \leq \frac{1}{v_{m}}\left|p_{m}(t)-p_{m}^{\star}\right|, \quad \forall i=1, \ldots, n .
$$

The upper-right Dini-derivative of $V(\boldsymbol{p}(t))$ along the trajectories of (1) is given by

$$
\begin{aligned}
D^{+} & V(\boldsymbol{p}(t)) \\
= & \frac{1}{v_{m}} \operatorname{sgn}\left(p_{m}(t)-p_{m}^{\star}\right) \dot{p}_{m}(t) \\
= & \frac{1}{v_{m}} \operatorname{sgn}\left(p_{m}(t)-p_{m}^{\star}\right) k_{m}\left(-p_{m}(t)+I_{m}(\boldsymbol{p}(t))\right) \\
= & k_{m}\left(-\frac{1}{v_{m}} \operatorname{sgn}\left(p_{m}(t)-p_{m}^{\star}\right)\left(p_{m}(t)-p_{m}^{\star}\right)\right) \\
& +k_{m}\left(\frac{1}{v_{m}} \operatorname{sgn}\left(p_{m}(t)-p_{m}^{\star}\right)\left(I_{m}(\boldsymbol{p}(t))-I_{m}\left(\boldsymbol{p}^{\star}\right)\right)\right) \\
\leq & k_{m}\left(-\frac{1}{v_{m}}\left|p_{m}(t)-p_{m}^{\star}\right|+\frac{1}{v_{m}}\left|I_{m}(\boldsymbol{p}(t))-I_{m}\left(\boldsymbol{p}^{\star}\right)\right|\right)
\end{aligned}
$$

where we have used the fact that $p_{m}^{\star}=I_{m}\left(\boldsymbol{p}^{\star}\right)$ to obtain the third equality. Since $\boldsymbol{I}(\boldsymbol{p})$ is c-contractive, it follows from Proposition 5 that

$$
\begin{aligned}
\frac{1}{v_{m}}\left|I_{m}(\boldsymbol{p}(t))-I_{m}\left(\boldsymbol{p}^{\star}\right)\right| & \leq\left\|\boldsymbol{I}(\boldsymbol{p}(t))-\boldsymbol{I}\left(\boldsymbol{p}^{\star}\right)\right\|_{\infty}^{\boldsymbol{v}} \\
& \leq c\left\|\boldsymbol{p}(t)-\boldsymbol{p}^{\star}\right\|_{\infty}^{\boldsymbol{v}} \\
& =\frac{c}{v_{m}}\left|p_{m}(t)-p_{m}^{\star}\right| .
\end{aligned}
$$

Combining (12) and (13) yields

$$
\begin{aligned}
D^{+} V(\boldsymbol{p}(t)) & \leq-k_{m}(1-c) \frac{1}{v_{m}}\left|p_{m}(t)-p_{m}^{\star}\right| \\
& =-k_{m}(1-c) V(\boldsymbol{p}(t)) \\
& \leq-k_{\min }(1-c) V(\boldsymbol{p}(t))
\end{aligned}
$$

where $k_{\min }=\min _{1<i<n} k_{i}$. Integrating both sides of (14) implies

$$
V(\boldsymbol{p}(t)) \leq V(\boldsymbol{p}(0)) e^{-k_{\min }(1-c) t}, \quad t \geq 0,
$$

which completes the proof.

Convergence time is a critical parameter in the evaluation of a distributed power control algorithm. Specifically, the convergence time $T_{\delta}$ of the algorithm (1) is defined to be the smallest $t \geq 0$ such that $\left\|\boldsymbol{p}(t)-\boldsymbol{p}^{\star}\right\|_{\infty}^{\boldsymbol{v}} \leq \delta$. If an interference function $\boldsymbol{I}(\boldsymbol{p})$ is c-contractive, then, according to Theorem 3

$$
\left\|\boldsymbol{p}(t)-\boldsymbol{p}^{\star}\right\|_{\infty}^{\boldsymbol{v}} \leq R_{0} e^{-k_{\min }(1-c) t},
$$

where $R_{0}=\left\|\boldsymbol{p}(0)-\boldsymbol{p}^{\star}\right\|_{\infty}^{\boldsymbol{v}}$. It follows that

$$
T_{\delta}=\frac{1}{k_{\min }(1-c)} \ln \frac{R_{0}}{\delta},
$$


and $\left\|\boldsymbol{p}(t)-\boldsymbol{p}^{\star}\right\|_{\infty}^{\boldsymbol{v}} \leq \delta$ for all $t \geq T_{\delta}$. We can see that the guaranteed convergence time of the continuous-time power control algorithm (1) goes to $\infty$ as $c$ tends to one.

The following theorem shows that power-control laws involving contractive interference functions are still exponentially stable when arbitrary bounded heterogeneous timevarying delays are introduced into (1). Moreover, it provides an explicit bound on the convergence rate of (3) that allows us to quantify the impact of the magnitude of the time delays on the convergence rate.

Theorem 4 (Delayed Power Control Algorithm): If an interference function $I: \mathbb{R}_{+}^{n} \rightarrow \mathbb{R}_{+}^{n}$ is c-contractive, then the unique fixed point $\boldsymbol{p}^{\star}$ of the continuous-time power control algorithm (3) is exponentially stable for any initial condition $\varphi(\cdot) \in \mathcal{C}_{++}$ and for any proportionality constant $k_{i}>0$. In particular

$$
\left\|\boldsymbol{p}(t)-\boldsymbol{p}^{\star}\right\|_{\infty}^{\boldsymbol{v}} \leq\left(\sup _{-\tau_{\max } \leq s \leq 0}\left\|\boldsymbol{\varphi}(s)-\boldsymbol{p}^{\star}\right\|_{\infty}^{\boldsymbol{v}}\right) e^{-k_{\min }(1-\bar{c}) t}
$$

holds for all $t \geq 0$, where $k_{\min }=\min _{1 \leq i \leq n} k_{i}$, and $\bar{c}$ is the unique positive solution to

$$
\bar{c}=c e^{k_{\min }(1-\bar{c}) \tau_{\max }} .
$$

Proof: Consider the following Lyapunov function

$$
V(\boldsymbol{p}(t))=\left\|\boldsymbol{p}(t)-\boldsymbol{p}^{\star}\right\|_{\infty}^{\boldsymbol{v}} .
$$

Similar to the proof of Theorem 3, taking the upper-right Diniderivative of $V(\boldsymbol{p}(t))$ along the trajectories of (3) yields

$$
\begin{aligned}
& D^{+} V(\boldsymbol{p}(t)) \\
& \leq k_{m}\left(-\frac{1}{v_{m}}\left|p_{m}(t)-p_{m}^{\star}\right|+\frac{1}{v_{m}}\left|I_{m}\left(\boldsymbol{p}^{d_{m}}(t)\right)-I_{m}\left(\boldsymbol{p}^{\star}\right)\right|\right) \\
& \leq k_{m}\left(-\frac{1}{v_{m}}\left|p_{m}(t)-p_{m}^{\star}\right|+c\left\|\boldsymbol{p}^{d_{m}}(t)-\boldsymbol{p}^{\star}\right\|_{\infty}^{\boldsymbol{v}}\right),
\end{aligned}
$$

where the index $m \in\{1, \ldots, n\}$ satisfies

$$
\frac{1}{v_{i}}\left|p_{i}(t)-p_{i}^{\star}\right| \leq \frac{1}{v_{m}}\left|p_{m}(t)-p_{m}^{\star}\right|, \quad \forall i=1, \ldots, n,
$$

and we have used the fact that $\boldsymbol{I}(\boldsymbol{p})$ is c-contractive to get the second inequality. Then

$$
\begin{aligned}
D^{+} & V(\boldsymbol{p}(t)) \\
& \leq k_{m}\left(-\left\|\boldsymbol{p}(t)-\boldsymbol{p}^{\star}\right\|_{\infty}^{\boldsymbol{v}}+c\left\|\boldsymbol{p}^{d_{m}}(t)-\boldsymbol{p}^{\star}\right\|_{\infty}^{\boldsymbol{v}}\right) \\
& \leq k_{m}\left(-V(\boldsymbol{p}(t))+c \max _{1 \leq i \leq n}\left\|\boldsymbol{p}\left(t-\tau_{i}^{m}(t)\right)-\boldsymbol{p}^{\star}\right\|_{\infty}^{\boldsymbol{v}}\right) \\
& =k_{m}\left(-V(\boldsymbol{p}(t))+c \max _{1 \leq i \leq n} V\left(\boldsymbol{p}\left(t-\tau_{i}^{m}(t)\right)\right)\right) \\
& \leq k_{m}\left(-V(\boldsymbol{p}(t))+c \max _{t-\tau_{\max } \leq s \leq t} V(\boldsymbol{p}(s))\right) .
\end{aligned}
$$

Since $V(\boldsymbol{p}) \geq 0$ and $0<c<1$, by applying Proposition 3, we obtain

$$
V(\boldsymbol{p}(t)) \leq\left(\sup _{-\tau_{\max } \leq s \leq 0} V(\boldsymbol{p}(s))\right) e^{-\eta_{m} t}, \quad t \geq 0,
$$

where $\eta_{m}$ is the unique positive solution of the equation

$$
\eta_{m}=k_{m}\left(1-c e^{\eta_{m} \tau_{\max }}\right) .
$$

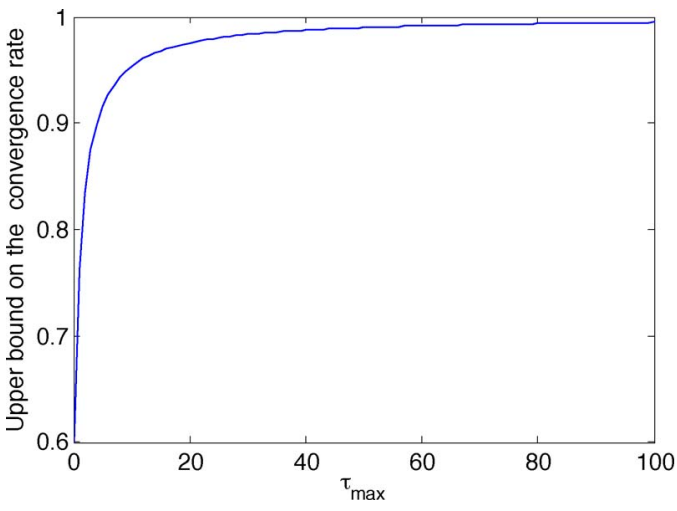

Fig. 1. Upper bound on the convergence rate $(\bar{c})$ with respect to the maximum delay bound $\tau_{\max } . \bar{c}$ is monotonically increasing with $\tau_{\max }$ and approaches one as $\tau_{\max }$ tends to infinity. In this example, $c=0.6, k_{\min }=1$ and $\tau_{\max }$ varies from 0 to 100 .

For each $i \in\{1, \ldots, n\}$, let $\eta_{i}$ be the unique positive solution to

$$
\eta_{i}=k_{i}\left(1-c e^{\eta_{i} \tau_{\max }}\right) .
$$

Since $\eta_{i}$, as a function of $k_{i}>0$, is monotonically increasing, we have $\eta \leq \eta_{i}$ for all $i$, where

$$
\eta=k_{\min }\left(1-c e^{\eta \tau_{\max }}\right) .
$$

This implies that $e^{-\eta_{m} t} \leq e^{-\eta t}$ for $t \geq 0$, and hence

$$
V(\boldsymbol{p}(t)) \leq\left(\sup _{-\tau_{\max } \leq s \leq 0} V(\boldsymbol{p}(s))\right) e^{-\eta t} .
$$

Letting $\bar{c}=1-\left(\eta / k_{\min }\right)$, the result follows immediately.

Although (16) does not admit an explicit solution, its solution $\bar{c}$ is always unique and satisfies

$$
c \leq \bar{c}<1 .
$$

Moreover, $\bar{c}$ is monotonically increasing with $\tau_{\max }$ and approaches one as $\tau_{\max }$ tends to infinity (see Fig. 1). Hence, while the power control law involving contractive interference functions remains exponentially stable for arbitrary bounded time-varying delays, the convergence rate deteriorates with increasing delays. Furthermore, the convergence time for the delayed continuous-time power control algorithm (3) is given by

$$
T_{\delta}=\frac{1}{k_{\min }(1-\bar{c})} \ln \frac{R_{0}}{\delta},
$$

where

$$
R_{0}=\sup _{-\tau_{\max } \leq s \leq 0}\left\|\boldsymbol{\varphi}(s)-\boldsymbol{p}^{\star}\right\|_{\infty}^{\boldsymbol{v}} .
$$

Thus, the guaranteed convergence time goes to $\infty$ as $\tau_{\max }$ tends to $\infty$.

Remark 4: In [15], it has been shown that discrete-time power control algorithms involving contractive interference functions converge exponentially to the unique fixed point, even in the presence of bounded time-varying delays. However, the extension of discrete-time results to the continuous-time 
domain is non-trivial. The reason for this is that discretetime power control iterations with contractive interference functions fall within a comprehensive framework, the Totally Asynchronous Algorithmic Model, for studying the stability of discrete-time systems with heterogeneous time-varying delays [22, Ch. 6]. To the best of our knowledge, however, there does not exist a corresponding framework for continuous-time systems with heterogeneous time-varying delays and, hence, the methodology used in this paper is completely different to the one used in [15]. Also note that our proof technique uses neither the Lyapunov-Krasovskii functional method nor the Lyapunov-Razumikhin method widely used to analyze the stability of continuous-time systems with time delays, since the Lyapunov-Krasovskii method often requires the time delay $\tau(t)$ to satisfy conservative conditions on its variation, and the Lyapunov-Razumikhin method often gives a sufficient condition for asymptotic stability. Our proof technique allows us not only to impose minimal restrictions on time-varying delays, but also to derive explicit exponential bounds on the decay rate of continuous-time power control algorithms involving contractive interference functions.

\section{The Foschini-Miljanic Algorithm}

While the Foschini-Miljanic (FM) algorithm falls within all three interference classes, neither the standard nor the two-sided scalable interference frameworks are able to guarantee exponential convergence rate and provide a guaranteed convergence time in the presence of heterogeneous time-varying delays. To show that the concept of contractive interference functions is useful for analyzing continuous-time power control algorithms, we consider the linear interference function described in (2). Equation (2) can be rewritten as

$$
I_{i}(\boldsymbol{p})=\sum_{j=1}^{n} m_{i j} p_{j}+\bar{\eta}_{i}, \quad i=1, \ldots, n,
$$

where $\bar{\eta}_{i}=\gamma_{i}\left(\eta_{i} / g_{i i}\right)$, and

$$
m_{i j}= \begin{cases}\gamma_{i} \frac{g_{i j}}{g_{i i}}, & j \neq i \\ 0, & j=i\end{cases}
$$

Define $M$ as an $n \times n$ matrix that has $m_{i j}$ as its elements. It is shown in [15] that if $\|M\|_{\infty}^{\boldsymbol{v}}<1$ for some $\boldsymbol{v}>\mathbf{0}$, the linear interference function is c-contractive with $c=\|M\|_{\infty}^{\boldsymbol{v}}$. In this case, the interference function has a unique fixed point and, according to Theorem 3, the associated continuous-time power control algorithm (1) is exponentially stable. That is, there exists a set of powers to which all transmitters converge exponentially fast, such that all transmitters meet their QoS requirements (minimum SINR for successful reception). This also coincides with the analysis in [2].

Since $M$ is a square non-negative matrix, according to Proposition $1, \rho(M)<1$ is a necessary and sufficient condition for the existence of a positive vector $\boldsymbol{v}$ for which $\|M\|_{\infty}^{\boldsymbol{v}}<1$. In this case, the following set of linear inequalities in $v$

$$
\begin{cases}M v & <\boldsymbol{v} \\ \boldsymbol{v} & >\mathbf{0}\end{cases}
$$

is feasible. According to Theorem 3, any feasible solution $v$ to the Linear Programming (LP) problem (19) ensures that the solution $\boldsymbol{p}(t)$ of the nominal continuous-time power control algorithm (1) is globally exponentially convergent to the unique fixed point $\boldsymbol{p}^{\star}$ with the convergence rate

$$
\alpha=k_{\min }\left(1-\|M\|_{\infty}^{\boldsymbol{v}}\right) .
$$

It is easily seen that the decay rate $\alpha$ depends on the choice of vector $\boldsymbol{v}$. Since $\rho(M) \leq\|M\|_{\infty}^{\boldsymbol{v}}$, we always have the following upper bound for the guaranteed convergence rate

$$
\alpha \leq \bar{\alpha}=k_{\min }(1-\rho(M)) .
$$

Note that if $M$ is irreducible, which is often a reasonable assumption (since we are not considering isolated groups of links that do not interact with each other), the positive right Perron-Frobenius eigenvector $\boldsymbol{v}_{\mathrm{PF}}$ of $M$ satisfies

$$
\rho(M)=\|M\|_{\infty}^{\boldsymbol{v}_{\mathrm{PF}}} .
$$

In this case, $\alpha=\bar{\alpha}$ and the solution $\boldsymbol{p}(t)$ of (1) satisfies

$$
\left\|\boldsymbol{p}(t)-\boldsymbol{p}^{\star}\right\|_{\infty}^{\boldsymbol{v}_{\mathrm{PF}}} \leq\left\|\boldsymbol{p}(0)-\boldsymbol{p}^{\star}\right\|_{\infty}^{\boldsymbol{v}_{\mathrm{PF}}} e^{-k_{\min }(1-\rho(M)) t}, \quad t \geq 0 .
$$

Therefore, the vector $\boldsymbol{v}_{\mathrm{PF}}$ gives the best convergence rate of the the nominal continuous-time power control algorithm (1) that our results can guarantee, provided that $M$ is irreducible.

We will now show that c-contractivity is a necessary and sufficient condition for exponential stability of the continuoustime Foschini-Miljanic algorithm with bounded heterogenous time-varying delays.

Theorem 5: Consider the continuous-time FM algorithm given by

$$
\frac{\mathrm{d} p_{i}(t)}{\mathrm{d} t}=k_{i}\left(-p_{i}(t)+\sum_{j=1}^{n} m_{i j} p_{j}\left(t-\tau_{j}^{i}(t)\right)+\bar{\eta}_{i}\right),
$$

where $m_{i j}$ and $\bar{\eta}_{i}$ are defined in (18). Let $M=\left[m_{i j}\right] \in \mathbb{R}^{n \times n}$. Then, the following statements are equivalent.

(a) $\rho(M)<1$.

(b) $\|M\|_{\infty}^{\boldsymbol{v}}<1$ for some $\boldsymbol{v}>\mathbf{0}$.

(c) The FM algorithm (20) is exponentially stable for any initial condition $\varphi(\cdot) \in \mathcal{C}_{++}$, for any proportionality constant $k_{i}>0$, and for all bounded heterogeneous timevarying delays.

Moreover, every solution $\boldsymbol{p}(t)$ of (20) satisfies

$$
\left\|\boldsymbol{p}(t)-\boldsymbol{p}^{\star}\right\|_{\infty}^{\boldsymbol{v}} \leq\left(\sup _{-\tau_{\max } \leq s \leq 0}\left\|\boldsymbol{\varphi}(s)-\boldsymbol{p}^{\star}\right\|_{\infty}^{\boldsymbol{v}}\right) e^{-k_{\min }(1-\bar{c}) t},
$$

where $\bar{c}$ is the unique positive solution to

$$
\bar{c}=\|M\|_{\infty}^{\boldsymbol{v}} e^{-k_{\min }(1-\bar{c}) \tau_{\max }} .
$$

Proof: $(a) \Leftrightarrow(b)$ : The matrix $M$ is non-negative. Hence, the equivalence of (a) and (b) follows directly from Proposition 1. 
$(b) \Rightarrow(c)$ : If $\|M\|_{\infty}^{\boldsymbol{v}}<1$ for some $\boldsymbol{v}>\mathbf{0}$, the linear interference functions is c-contractive with $c=\|M\|_{\infty}^{\boldsymbol{v}}[15$, Th. 1]. It now follows from Theorem 4 that (b) implies (c).

$(c) \Rightarrow(b)$ Assume that (20) is exponentially stable for any arbitrary bounded time delays. Particularly, let $\tau_{\max }=0$. Then, the following linear system is exponentially stable

$$
\dot{\boldsymbol{x}}(t)=K\left(-I_{n}+M\right) \boldsymbol{x}(t),
$$

where $K=\operatorname{diag}\left(k_{1}, \ldots, k_{n}\right)$, and $I_{n}$ is the $n \times n$ identity matrix. It follows that $K\left(-I_{n}+M\right)$ is a Hurwitz matrix, i.e., all its eigenvalues have negative real parts. Moreover, $-I_{n}+M$ is a Metzler matrix, since $M$ is non-negative. Note that for Metzler matrices, Hurwitz stability is equivalent to diagonal stability [31, Th. 2.2.1]. More precisely, $K\left(-I_{n}+M\right)$ is stable if and only if $-I_{n}+M$ is stable. Therefore, the linear system $\dot{\boldsymbol{x}}(t)=\left(-I_{n}+M\right) \boldsymbol{x}(t)$ is also exponentially stable. Since $-I_{n}+M$ is Metzler and Hurwitz, there is a vector $\boldsymbol{v}>\mathbf{0}$ such that $\left(-I_{n}+M\right) \boldsymbol{v}<\mathbf{0}$ [32, Proposition 2], which implies that $M \boldsymbol{v}<\boldsymbol{v}$. Therefore, $\|M\|_{\infty}^{\boldsymbol{v}}<1$. This completes the proof of the theorem.

As shown in Theorem 5, the FM algorithm is exponentially stable for all bounded heterogeneous time-varying delays if and only if the LP problem (19) is feasible. Moreover, any vector $\boldsymbol{v}$ satisfying (19) can be used to find a guaranteed convergence rate by computing the associated $\bar{c}$ in (21). Equation (21) has three parameters: the maximum delay bound $\tau_{\max }$, the positive vector $\boldsymbol{v}$, and $\bar{c}$. For any fixed $\tau_{\max } \geq 0$, and any fixed $\boldsymbol{v}>$ $\mathbf{0}$, (21) is a nonlinear equation with respect to $\bar{c}$. Clearly, the exponential bound on the decay rate that Theorem 5 can provide depends on the choice of vector $\boldsymbol{v}$, and that an arbitrary feasible $v$ not necessarily gives a tight bound on the actual decay rate. For any fixed $\tau_{\max } \geq 0$, since $\bar{c}$ is monotonically increasing in $\|M\|_{\infty}^{\boldsymbol{v}}$, we have $\bar{c}^{\star} \leq \bar{c}$, where $\bar{c}^{\star}$ is the unique solution to

$$
\bar{c}^{\star}=\rho(M) e^{-k_{\min }\left(1-\bar{c}^{\star}\right) \tau_{\max }} .
$$

Hence, the convergence rate of (20) is always upper bounded by

$$
\alpha \leq \bar{\alpha}=k_{\min }\left(1-\bar{c}^{\star}\right),
$$

which implies that if $M$ is irreducible, the positive right PerronFrobenius eigenvector $\boldsymbol{v}_{\mathrm{PF}}$ of $M$ also gives the best convergence rate of the delayed FM algorithm that our bound can guarantee.

Remark 5: The asymptotic stability of the FM algorithm was investigated for constant delays in [5] using the multivariate Nyquist criterion [33], and for bounded time-varying delays in [7] within the context of positive linear systems. Similar to Theorem 5, the stability conditions presented in these works are necessary and sufficient, but for asymptotic stability. In this paper, however, we show that the condition for exponential stability is also necessary and sufficient. Furthermore, the impact of delays on the convergence rate of (20) has been missing in [5], [7], whereas Theorem 5 provides an explicit bound on the convergence rate that allows us to quantify the impact of delays on the convergence rate.

\section{NumERICAL EXAMPLES}

In this section, we demonstrate the validity of our analytical results and illustrate the accuracy of our guaranteed bounds on the convergence rate of continuous-time power control algorithms. We first consider a linear power control algorithm-the FM algorithm [2] — and include $(i)$ an example for which the algorithm converges for bounded time-varying delays to show that our convergence rate bound accurately captures the true system behavior; and (ii) an example for which the linear interference function does not satisfy $\rho(M)<1$ and hence diverges, to justify that the derived condition is necessary and sufficient. Next, we consider a nonlinear power control algorithm — the UBPC algorithm [20] — in a scenario where the algorithm converges for bounded time-varying delays. We explore the impact of the maximum delay bound on the system response and on the accuracy of our decay rate bound and find that the guaranteed bound estimates the true system dynamics well, despite that the analysis of this case is only sufficient.

\section{A. Linear Interference Function}

We consider a wireless network with 4 users, characterized by the matrix

$$
G=\left[\begin{array}{llll}
0.4000 & 0.0082 & 0.0419 & 0.0579 \\
0.0160 & 0.8530 & 0.0424 & 0.0043 \\
0.0200 & 0.0017 & 0.1405 & 0.0010 \\
0.1030 & 0.0036 & 0.0104 & 0.4050
\end{array}\right] \times 10^{-3} .
$$

For this example, the SINR threshold and the thermal noise for each node is set to $\gamma_{i}=3$ and $\eta_{i}=0.04 \mathrm{mWatts}$, respectively. The initial power $p_{i}(0)$ for all users is set to $1 \mathrm{mWatt}$. The timevarying delays have, somewhat arbitrarily, been set to evolve as $\tau(t)=0.5 \tau_{\max }+0.5 \tau_{\max } \sin (t)$. We consider an implementation of the Foschini-Miljanic algorithm, with proportionality constant $k_{i}=0.8$. It is easy to verify that the spectral radius of the network is $\rho(M)=0.7146<1$, where the matrix $M$ is defined in (18). Therefore, according to Theorem 5, the FM algorithm with bounded time-varying delays converges exponentially to the unique fixed point. A simulation of the network characterized by the matrix (22) show that SINRs indeed converge to the desired SINR and power levels to the minimal power vector; see Fig. 3 .

The theoretical upper bounds on the decay rate of the FM algorithm as obtained in Theorem 5 have been compared with the actual decay rate of the FM algorithm for the wireless network characterized by the matrix (22). Since the delays are timevarying and usually smaller than the maximum bound, there is a gap between the theoretical and the actual decay rates that one observes in simulations, see Fig. 2. As $M$ is irreducible, $\|M\|_{\infty}^{\boldsymbol{v}}=\rho(M)=0.7146$. For this network, we then have numerically calculated the solution to the (21) for the values of maximum delay bounds we consider in the simulations and we have $\bar{c}_{5}=0.9332, \bar{c}_{20}=0.9802$ and $\bar{c}_{40}=0.9898$. We observe that when time delays are introduced, $\bar{c}$ increases monotonically with $\tau_{\max }$, meaning that the convergence rate decreases.

We now increase the SINR threshold to $\gamma_{i}=4.25$, while keeping all the other parameters fixed. The spectral radius of 


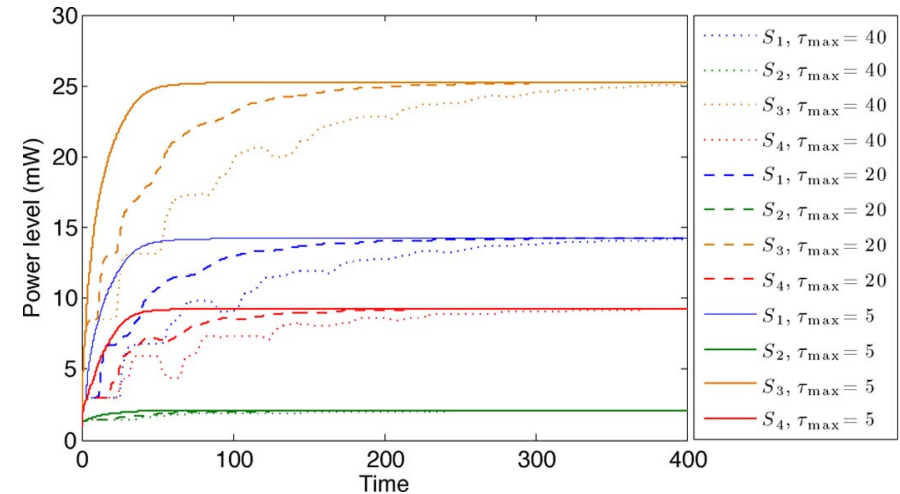

Fig. 2. Convergence of the FM algorithm with desired SINR $\gamma_{i}=3$, thermal noise $\eta=0.04 \mathrm{mWatts}$ and proportionality gain $k_{i}=0.8$ for all users. The solid, dashed and dotted lines illustrate the convergence of the algorithm in the presence of delays with maximum delay bound 5,20 and 40, respectively.

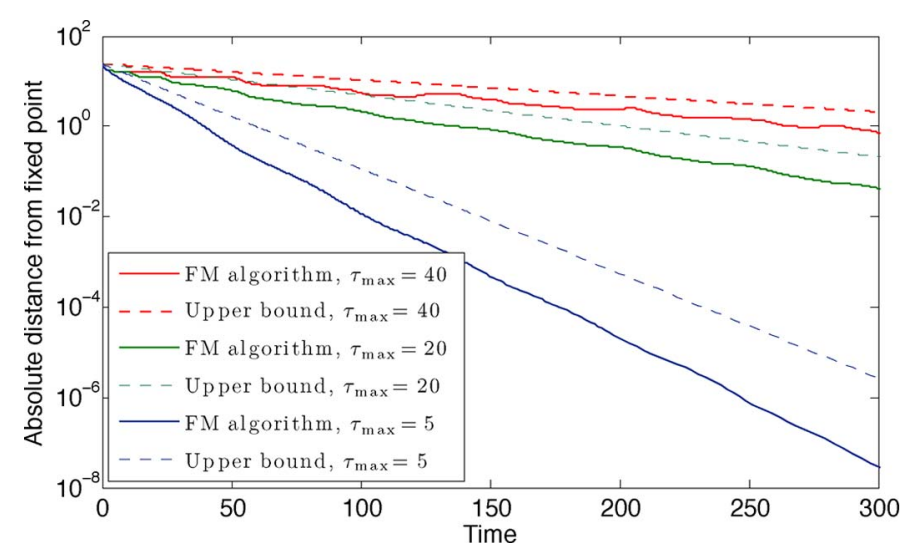

Fig. 3. Comparison of upper bound on the convergence rate of the FM algorithm as obtained in Theorem 5 and the actual convergence rate of the FM algorithm for the wireless network characterized by matrix (22), for $\tau_{\max }=5,20$ and 40 . The dashed lines show the theoretical upper bound on the convergence rate, while the solid lines show the actual convergence rate of the FM algorithm.

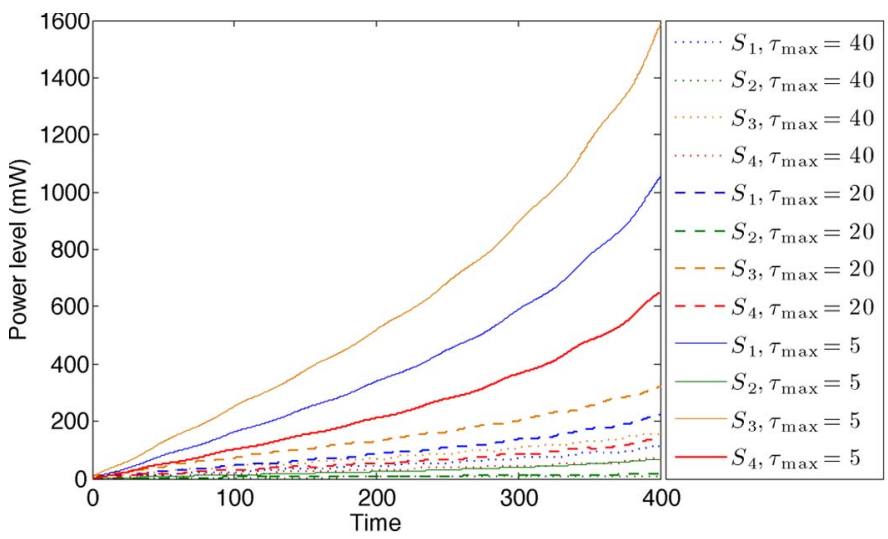

Fig. 4. Divergence of the FM algorithm with desired SINR $\gamma_{i}=4.25$, thermal noise $\eta=0.04 \mathrm{mWatts}$ and proportionality gain $k_{i}=0.8$ for all users. The solid, dashed and dotted lines illustrate the divergence of the algorithm in the presence of delays with maximum delay bound 5, 20 and 40, respectively.

the network becomes $\rho(M)=1.0123>1$ and the power levels diverge, see Fig. 4. This example justifies that the stability condition is both necessary and sufficient.

\section{B. Nonlinear Interference Function}

Next, we consider a continuous-time implementation of the Utility-Based Power Control (UBPC) algorithm from [20], whose the associated interference function is

$$
I_{i}^{u}(\mathbf{p})=\left(\frac{\sum_{j \neq i} g_{i j} p_{j}+\eta_{r}}{g_{i i}}\right) f_{i}^{-1}\left(\alpha_{i} \frac{\sum_{j \neq i} g_{i j} p_{j}+\eta_{r}}{g_{i i}}\right),
$$

where $i=1, \ldots, n$, and $\alpha_{i}$ is a price coefficient. Here, $f_{i}\left(S I R_{i}\right)=U_{i}^{\prime}\left(S I R_{i}\right)$ in the concave part of $U_{i}$ where $U_{i}$ is a utility function of user $i$. In their paper, Xiao et al. use a sigmoidal utility function

$$
U_{i}\left(S I R_{i}\right)=\frac{1}{1+e^{-a_{i}\left(S I R_{i}-b_{i}\right)}},
$$

where

$$
b_{i}=\gamma_{i}-a_{i}^{-1} \ln \left(a_{i} \gamma_{i}-1\right) .
$$

Let us define $M^{b}=\left[m_{i j}^{b}\right]$ to be

$$
m_{i j}^{b}= \begin{cases}b_{i} \frac{g_{i j}}{g_{i i}}, & j \neq i \\ 0, & j=i .\end{cases}
$$

In [15], it is shown that if $c=\rho\left(M^{b}\right)<1$, then $I^{u}$ is a c-contractive interference function. We will next show that Theorem 4 provides an explicit bound on the decay rate of UBPC algorithm that allows us to analytically quantify the impact of delays on the convergence rate of the algorithm.

Again, we consider a wireless network with 4 users, characterized by matrix (26),

$$
G=\left[\begin{array}{llll}
0.5930 & 0.0559 & 0.0416 & 0.0419 \\
0.0189 & 0.4860 & 0.0160 & 0.0266 \\
0.0199 & 0.0407 & 0.1890 & 0.0267 \\
0.0699 & 0.0499 & 0.0541 & 0.4950
\end{array}\right] \times 10^{-3} .
$$

The SINR threshold for each node is set to $\gamma_{i}=3$ and the thermal noise is given by $\eta_{i}=0.004 \mathrm{mWatts}$. The parameter $\alpha_{i}$ is set to 1 . The time-varying delays have been also in this case been simulated using $\tau(t)=0.5 \tau_{\max }+0.5 \tau_{\max } \sin (t)$. We assume that four users in the system use sigmoidal utility function with $a_{i}=5000$, and $b_{i}=2.998$ is found according to (25). Since $\rho\left(M^{b}\right)=0.81244<1$, according to Theorem 4, the UBPC algorithm with bounded time-varying delays converges exponentially to the unique fixed point. A simulation of the network characterized by the matrix (26) show that SINRs converge to the desired SINR and power levels to the minimal power vector; see Fig. 5.

The theoretical upper bounds on the decay rate of the UBPC algorithm as obtained in Theorem 4 have been compared with the actual decay rate of the UBPC algorithm for the wireless network characterized by the matrix (26), see Fig. 6 . For this network, we have numerically calculated $\bar{c}$ in (16) for the values of maximum delay bounds we consider in the simulations and we have $\bar{c}_{5}=0.95863, \bar{c}_{20}=0.98778$ and $\bar{c}_{40}=0.99371$. 


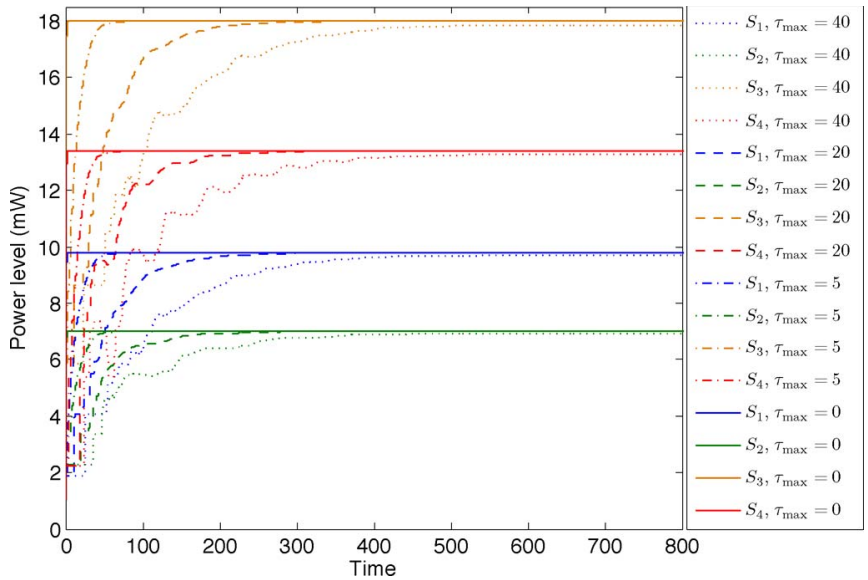

Fig. 5. Convergence of the UBPC algorithm with desired SINR $\gamma_{i}=1.5$, thermal noise $\eta=0.04 \mathrm{mWatts}$ and proportionality gain $k_{i}=0.1$ for all users. The solid, dashed, dot-dashed and dotted lines illustrate the convergence of the algorithm in the presence of delays with maximum delay bound $0,5,20$ and 40 , respectively.

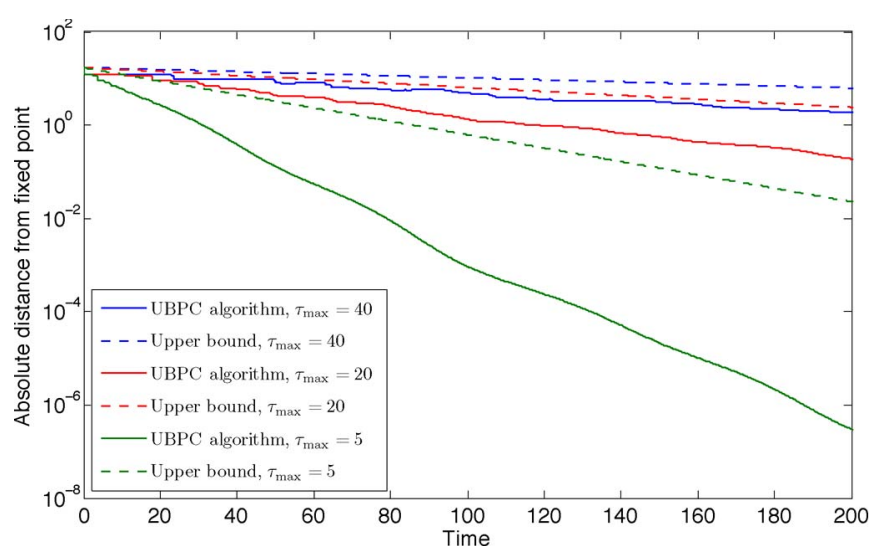

Fig. 6. Comparison of upper bound on the decay rate of the UBPC algorithm and the actual decay rate of the UBPC algorithm for the wireless network characterized by matrix (26), for $\tau_{\max }=5,20$ and 40 . The dashed lines show the theoretical upper bound on the decay rate, while the solid lines show the actual decay rate of the UBPC algorithm.

\section{CONCLUSIONS}

This paper considered the dynamics of continuous-time power control laws under an anthology of important classes of interference functions. We developed a comprehensive stability analysis framework that accounts for bounded heterogeneous time-varying delays. The first set of results established global asymptotic stability of power control laws involving two-sided scalable interference functions, and include previous work on standard interference functions as a special case. Next, we proved global exponential stability of power control laws involving contractive interference functions and derived explicit bounds on the decay rate which allows to, for the first time, quantify the impact of delays on the rate of convergence of the transmit powers. For the case of linear interference functions, we demonstrated that our results were necessary and sufficient. Illustrative examples demonstrated the validity of our theoretical results.

\section{ACKNOWLEDGMENT}

We would like to thank the Associate Editor and the anonymous reviewers for suggestions that helped to improve the quality of the paper.

\section{REFERENCES}

[1] J. Zander, "Distributed co-channel interference control in cellular radio systems," IEEE Trans. Vehic. Technol., vol. 41, no. 3, pp. 305-311, Aug. 1992.

[2] G. J. Foschini and Z. Miljanic, "A simple distributed autonomous power control algorithm and its convergence," IEEE Trans. Vehic. Technol., vol. 42, pp. 641-646, 1993.

[3] M. M. Olama, S. M. Djouadi, and C. D. Charalambous, "A general framework for continuous-time power control in time-varying long term fading wireless networks," in Proc. 9th IASTED Int. Conf. Control and Applications, 2007, pp. 69-74.

[4] W.-C. Su, B.-H. Jung, S.-Y. Chang, and Z. Gajic, "Unified continuous- and discrete-time uplink power control problem formulation for SIR-based wireless networks," in Proc. EEE Sarnoff Symp., Apr. 2009, pp. 1-5.

[5] T. Charalambous, I. Lestas, and G. Vinnicombe, "On the stability of the Foschini-Miljanic algorithm with time-delays," in CDC, 2008, pp. 2991-2996.

[6] T. Charalambous and Y. Ariba, "On the stability of a power control algorithm for wireless networks in the presence of time-varying delays," in Proc. 10th European Control Conf. (ECC), Aug. 2009.

[7] A. Zappavigna, T. Charalambous, and F. Knorn, "Unconditional stability of the Foschini-Miljanic algorithm," Automatica, vol. 48, no. 1, pp. 219224, 2012.

[8] I. Lestas, "Power control in wireless networks: Stability and delay independence for a general class of distributed algorithms," IEEE Trans. Autom. Control, vol. 57, no. 5, pp. 1253-1258, May 2012.

[9] D. Mitra, "An asynchronous distributed algorithm for power control in cellular radio systems," in Proc. 4th WINLAB Workshop, New Brunswick, NJ, 1993, Rutgers University.

[10] R. Yates, "A framework for uplink power control in cellular radio systems," IEEE J. Select. Areas Communi., vol. 13, no. 7, pp. 1341-1347, 1995.

[11] C. Sung and K. Leung, "A generalized framework for distributed power control in wireless networks," IEEE Trans. Inform. Theory, vol. 51, no. 7, pp. 2625-2635, 2005.

[12] A. Moller and U. Jonsson, "Stability of high order distributed power control," in Proc. 48th IEEE Conf. Decision and Control, Dec. 2009, pp. 4963-4970.

[13] A. Moller and U. Jonsson, "Input-output analysis of power control in wireless networks," IEEE Trans. Autom. Control, vol. 58, no. 4, pp. 834$846,2013$.

[14] H. Boche and M. Schubert, "A unifying approach to interference modeling for wireless networks," IEEE Trans. Signal Process., vol. 58, no. 6, pp. 3282-3297, Jun. 2010.

[15] H. Feyzmahdavian, M. Johansson, and T. Charalambous, "Contractive interference functions and rates of convergence of distributed power control laws," IEEE Trans. Wireless Commun., vol. 11, no. 12, pp. 4494-4502, Dec. 2012.

[16] T. Alpcan, T. Başar, R. Srikant, and E. Altman, "CDMA uplink power control as a noncooperative game," Wireless Netw., vol. 8, pp. 659-670, 2002.

[17] E. Altman and Z. Altman, "S-modular games and power control in wireless networks," IEEE Trans. Autom. Control, vol. 48, no. 5, pp. 839-842, May 2003.

[18] T. Alpcan, T. Basar, and S. Dey, "A power control game based on outage probabilities for multi-cell wireless data networks," IEEE Trans. Wireless Commun., vol. 5, no. 4, pp. 890-899, Apr. 2006.

[19] X. Fan, T. Alpcan, M. Arcak, T. J. Wen, and T. Basar, "A passivity approach to game-theoretic CDMA power control," Automatica, vol. 42, no. 11 , pp. $1837-1847,2006$.

[20] M. Xiao, N. Shroff, and E. Chong, "A utility-based power-control scheme in wireless cellular systems," IEEE J. Select. Areas Commun., vol. 11, no. 2, pp. 210-221, 2003.

[21] A. Berman and R. J. Plemmons, Nonnegative Matrices in Mathematical Sciences. New York: Academic Press, 1979.

[22] D. P. Bertsekas and J. N. Tsitsiklis, Parallel and Distributed Computation. Upper Saddle River, NJ: Prentice-Hall, 1989.

[23] R. D. Driver, Ordinary and Delay Differential Equations. New York: Springer, 1977. 
[24] C. W. Sung and K. K. Leung, "Opportunistic power control for throughput maximization in mobile cellular systems," in Proc. IEEE Int. Conf. Communications (ICC), 2004, vol. 5, pp. 2954-2958.

[25] K. K. Leung and C. W. Sung, "An opportunistic power control algorithm for cellular network," IEEE/ACM Trans. Netw., vol. 14, no. 3, pp. 470478,2006

[26] H. Y. Kwan, C. W. Sung, K. K. Leung, and K. W. Shum, "Opportunistic power control with rate adaptation for video conferencing services," in Proc. IEEE Int. Conf. Communications (ICC), 2006, vol. 12, pp. 5331-5335.

[27] W. M. Haddad, V. Chellaboina, and Q. Hui, Nonnegative and Compartmental Dynamical Systems. Princeton, NJ: Princeton, 2010.

[28] L. Farina and S. Rinaldi, Positive Linear Systems: Theory and Applications. New York: Wiley, 2000.

[29] J. R. Haddock and J. Terjeki, "Lyapunov-Razumikhin functions and invariance principle for functional differential equations," J. Differ. Equat., vol. 48, pp. 95-122, 1983.

[30] E. N. Chukwu, Stability and Time-Optimal Control of Hereditary Systems. New York: Academic Press, 1992, ser. Mathematics in Science and Engineering.

[31] E. Kaszkurewicz and A. Bhaya, Matrix Diagonal Stability in Systems and Computation. Boston, MA: Birkhauser, 2000.

[32] A. Rantzer, "Distributed control of positive systems," in Proc. 50th IEEE Conf. Decision and Control and European Control Conf. (CDC-ECC), 2011, pp. 6608-6611.

[33] C. A. Desoer and Y. Yang, "On the generalized Nyquist stability criterion," IEEE Trans. Autom. Control, vol. 25, no. 1, pp. 187-196, 1980.

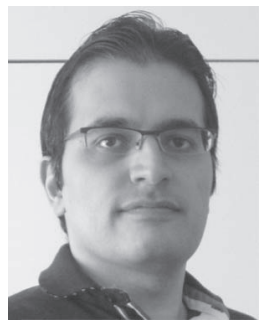

Hamid Reza Feyzmahdavian received the B.S. and M.Sc. degrees in electrical engineering with specialization in automatic control from Sharif University of Technology, Tehran, Iran. Since 2011, he has been working toward the Ph.D. degree at the Department of Automatic Control, KTH Royal Institute of Technology, Stockholm, Sweden.

His research interests include positive systems, distributed control and optimization, wireless networking and time-delay systems.

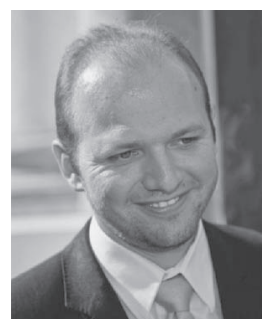

Themistoklis Charalambous received the B.A. and M.Eng. degrees in electrical and information sciences from Cambridge University, Cambridge, U.K., in 2005. He pursued his Ph.D. in the Control Laboratory, of the Engineering Department, Cambridge University in 2009.

$\mathrm{He}$ worked as a Research Associate at Imperial College London and as a Visiting Lecturer at the Department of Electrical and Computer Engineering, University of Cyprus. He is currently working at the Automatic Control Lab of the School of Electrical Engineering at the Royal Institute of Technology (KTH) as a Research Associate. His research involves cooperative control, distributed decision making, game theory, and control to various resource allocation problems in complex and networked systems, such as wireless ad hoc networks.

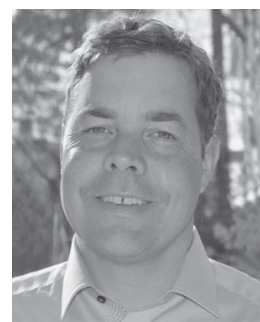

Mikael Johansson received the M.Sc. and Ph.D. degrees in electrical engineering from the University of Lund, Sweden, in 1994 and 1999, respectively.

He held postdoctoral positions at Stanford University and UC Berkeley before joining KTH in 2002, where he now serves as full professor. His research interests are in distributed optimization, wireless networking, and control. He has published one book and more than 100 papers, several of which are ISIhighly cited.

Dr. Johansson has served on the editorial board of Automatica and on the program committee for conferences such as IEEE CDC, IEEE Infocom, ACM SenSys, and played a leading role in several national and international research projects in control and communications. 